\title{
Dynamic near-infrared spectroscopy assessment as an important tool to explore pulmonary arterial hypertension pathophysiology
}

\section{To the Editor:}

With great interest we read the recent article by PANAGIOTOU et al. [1] in which the authors investigated the correlation between tissue oxygen measurements $\left(\mathrm{StO}_{2}\right)$ at rest and during constant-workload exercise with the analogous measurements of mixed venous oxygen saturation at the level of pulmonary artery $\left(\mathrm{SvO}_{2}\right)$ and inferior vena cava in patients with pulmonary arterial hypertension (PAH). In this study, the authors used near-infrared spectroscopy (NIRS) to assess the vastus lateralis muscle tissue oxygenation during exercise.

Although limited by the number of patients (particularly those with measurements during exercise $(n=10)$ ), the results of this study showed that $\mathrm{StO}_{2}$ had a moderate correlation with $\mathrm{SvO}_{2}$ at rest and a good correlation during exercise. This study confirms the significant contribution of peripheral muscle alterations to the exercise intolerance in PAH patients.

Examining further the explanatory mechanisms of $\mathrm{SvO}_{2}$ decrease during exercise, it would be interesting (if data are available) for the authors to provide information on cardiac output and arteriovenous oxygen content difference measurements to configure the oxygen extraction rate during exercise. This element would possibly add valuable insights into the study, although the exercise protocol studied was submaximal and there was no healthy control group. This limitation of the study might possibly misdiagnose oxygen extraction rate impairment in PAH patients, as this varies according to the level of exercise (maximal or submaximal). An impaired oxygen extraction rate during maximal exercise has been reported by TolLE et al. [2], partially confirming the peripheral muscle hypothesis. Another recent study [3] has also shown the presence of greater changes in myoglobin-deoxyhaemoglobin level and lower tissue oxygenation index during normoxic submaximal exercise protocol in patients with pulmonary hypertension compared with controls.

In a previous study from our institute investigating the peripheral muscle pathophysiology in PAH patients [4], we dynamically assessed the peripheral muscle microcirculation with NIRS methodology using the 3-min brachial artery occlusion technique before, during and after $15 \mathrm{~min}$ of breathing $100 \%$ of oxygen. Interestingly, we found that these patients had a lower $\mathrm{StO}_{2}$ at rest (mean $\pm \mathrm{SD}$ 65.8 $\pm 14.9 \%$ versus $82.1 \pm 4.0 \%$, $\mathrm{p}<0.005)$, a trend toward a lower oxygen consumption rate during occlusion $(35.3 \pm 9.1 \%$ versus $43.4 \pm 19.7 \%$ per $\min )$ and a significant higher hyperaemia reactive time $(3.0 \pm 0.6$ versus $2.0 \pm 0.3 \mathrm{~min}, \mathrm{p}<0.001)$ after the release of the occlusion (an index of endothelial function) compared to the healthy control group.

Following the results of that study, we have considered that these findings reflect a skeletal muscle mismatch of regional tissue perfusion and oxidative metabolism in $\mathrm{PAH}$ patients. Although this phenomenon needs further investigation for explanatory mechanisms, it could be, to some degree, explained by the previously reported imbalance of autonomic nervous system activity in PAH [5], the pronounced increased sympathetic overactivity of local and systemic neurohumoral factors, and the anatomical and functional skeletal muscle alterations (muscle atrophy and mitochondrial dysfunction) [6] as the main mediators.

A further interesting finding of our study [4] was that hyperoxic breathing even after only 15 min was sufficient to demonstrate a marked further increase to hyperaemia time after the release of vascular occlusion ( $3.0 \pm 0.6$ to $4.2 \pm 0.7 \mathrm{~min}, \mathrm{p}<0.007)$, indicating important endothelial dysfunction effects of oxygen

@ERSpublications

Near-infrared spectroscopy: a useful methodology for pulmonary arterial hypertension http://ow.ly/7wwQ3065Cpb

Cite this article as: Dimopoulos S, Tzanis G, Karabinis A, et al. Dynamic near-infrared spectroscopy assessment as an important tool to explore pulmonary arterial hypertension pathophysiology. Eur Respir J 2017; 49: 1601932 [https://doi.org/10.1183/13993003.01932-2016]. 
therapy application possibly due to increased oxidative stress and evoked vasoconstriction. This was a remarkable finding taking into account the potential deleterious effects of oxygen therapy in PAH patients.

We strongly believe that the above findings merit further investigation to add to the exploration of the explanatory mechanisms of PAH pathophysiology.

NIRS during exercise and, in particular, during the occlusion technique is a simple, dynamic, noninvasive methodology that can offer useful information for research purposes, monitoring and prognosis, and possibly contribute to assessing the possible beneficial effects of different treatment strategies including current and novel pulmonary vasodilators, oxygen therapy targets, and exercise training in patients with PAH.

Stavros Dimopoulos ${ }^{1,2,3}$, Giorgos Tzanis ${ }^{1,2}$, Andreas Karabinis ${ }^{3}$ and Serafim Nanas ${ }^{1,2}$

${ }^{1}$ Cardiopulmonary Exercise Testing and Rehabilitation Laboratory, National and Kapodistrian University of Athens, Athens, Greece. ${ }^{2} 1$ st Critical Care Medicine Dept, Evaggelismos Hospital, Athens, Greece. ${ }^{3}$ Cardiothoracic Intensive Care Unit, Onassis Cardiac Surgery Center, Athens, Greece.

Correspondence: Stavros Dimopoulos, Cardiopulmonary Exercise Testing and Rehabilitation Laboratory, National and Kapodistrian University of Athens, 45-47 Ypsilantou str, 10676 Athens, Greece. E-mail: a-icu@med.uoa.gr

Received: Oct 032016 | Accepted: Oct 082016

Conflict of interest: None declared.

\section{References}

1 Panagiotou M, Vogiatzis I, Louvaris Z, et al. Near infrared spectroscopy for the assessment of peripheral tissue oxygenation in pulmonary arterial hypertension. Eur Respir J 2016; 48: 1224-1227.

2 Tolle J, Waxman A, Systrom D. Impaired systemic oxygen extraction at maximum exercise in pulmonary hypertension. Med Sci Sports Exerc 2008; 40: 3-8.

3 Malenfant S, Potus F, Mainguy V, et al. Impaired skeletal muscle oxygenation and exercise tolerance in pulmonary hypertension. Med Sci Sports Exerc 2015; 47: 2273-2282.

4 Dimopoulos S, Tzanis G, Manetos C, et al. Peripheral muscle microcirculatory alterations in patients with pulmonary arterial hypertension: a pilot study. Respir Care 2013; 58: 2134-2141.

5 Dimopoulos S, Anastasiou-Nana M, Katsaros F, et al. Impairment of autonomic nervous system activity in patients with pulmonary arterial hypertension: a case control study. J Card Fail 2009; 15: 882-889.

6 Manders E, Rain S, Bogaard HJ, et al. The striated muscles in pulmonary arterial hypertension: adaptations beyond the right ventricle. Eur Respir J. 2015; 46: 832-842.

From the authors:

We thank S. Dimopoulos and co-workers for their particular interest in our study [1] and their contributions to this issue. The peripheral muscle hypothesis in pulmonary arterial hypertension (PAH) [2] is certainly of great and growing interest due to the potential of muscle function as a target for meaningful intervention. Accordingly, our study explored the value of quadriceps muscle oxygenation profiles in patients with PAH by means of near-infrared spectroscopy (NIRS) [1]. The satisfactory correlation between vastus lateralis muscle tissue oxygenation index $\left(\mathrm{StO}_{2}\right)$ and mixed venous oxygen saturation $\left(\mathrm{SvO}_{2}\right)$ both at rest and during exercise support the use of NIRS in the noninvasive investigation of patients with PAH. Importantly, they suggest that skeletal muscle oxygenation profiles reflect the pathophysiology of PAH.

We know that factors determining local muscle oxygenation are modulated by the rate of oxygen delivery and of oxygen extraction [3]. Exploration of the relative contribution of oxygen delivery and oxygen extraction in peripheral muscle $\mathrm{StO}_{2}$ in $\mathrm{PAH}$ would be of paramount significance, as it would help to clarify whether muscle dysfunction in PAH is a mere consequence of impaired central haemodynamics, or whether it is due to a primary, intrinsic myopathy [4]. To this end, application of NIRS technology during

@ERSpublications

Near-infrared spectroscopy offers a qualitative, noninvasive indication of mixed venous oxygen saturation in PAH http://ow.ly/MctV306eEYC

Cite this article as: Panagiotou M, Vogiatzis I, Louvaris Z, et al. Dynamic near-infrared spectroscopy assessment as an important tool to explore pulmonary arterial hypertension pathophysiology. Eur Respir J 2017; 49: 1602161 [https://doi.org/10.1183/13993003.02161-2016]. 
exercise and/or in combination with other methods, such as the occlusion technique [5], the indocyanine green dye technique [6] and histological examination, offers a very promising opportunity. Indocyanine green dye, which has long been used for the assessment of cardiac output and plasma volume, is detectable by NIRS and has been used in this way to measure regional blood flow in respiratory and locomotor muscles [7-9].

Despite the fact that our study was limited by design in the investigation of muscle function (including the use of supplementary oxygen and submaximal exercise protocol, and the absence of a control group), when prompted by S. Dimopoulos and co-workers we proceeded to further interrogation of our data. Calculations in the exercising patient group $(n=10)$ were unfortunately not feasible due to missing data; however, interrogation of complete patient resting data sets $(n=25)$ revealed some interesting results.

We calculated the estimated systemic oxygen delivery $\left(\mathrm{DO}_{2}\right)$, the product of cardiac output and arterial oxygen content, the latter term being calculated as the product of $1.34 \times$ haemoglobin concentration $\times \mathrm{SpO}_{2}$ (percentage arterial oxygen saturation measured by pulse oximetry). The systemic arteriovenous oxygen content difference (a- $\mathrm{vO}_{2}$ diff) was calculated by dividing oxygen uptake by cardiac output (the Fick principle) whereas the systemic oxygen extraction rate was calculated as the ratio of $\mathrm{a}-\mathrm{vO}_{2}$ diff to arterial oxygen content [10].

Resting oxygen content (in $\mathrm{mL} \cdot \mathrm{dL}^{-1}$ ) was $17.2 \pm 2.9, \mathrm{DO}_{2}$ (in $\mathrm{mL} \cdot \mathrm{min}^{-1}$ ) was $794 \pm 317$, a- $\mathrm{vO}_{2}$ diff (in $\mathrm{mLO}_{2} \cdot \mathrm{dL}^{-1}$ ) was $5.7 \pm 2.2$ and oxygen extraction rate (\%) was $34 \pm 13$. These results do not diverge significantly from normality and do not suggest an overt skeletal muscle dysfunction at rest; however, they cannot exclude an underlying muscle impairment that may become clinically significant during exercise.

Importantly, resting $\mathrm{StO}_{2}$ correlated positively with $\mathrm{DO}_{2}(\mathrm{r}=0.556, \mathrm{p}=0.004)$ and inversely with oxygen extraction rate $(\mathrm{r}=-0.695, \mathrm{p}<0.001)$. In a similar fashion, resting $\mathrm{SvO}_{2}$ also correlated positively with $\mathrm{DO}_{2}(\mathrm{r}=0.761, \mathrm{p}<0.001)$ and inversely with oxygen extraction rate $(\mathrm{r}=-0.980, \mathrm{p}<0.001)$. These novel findings are important because a) they confirm a positive correlation of peripheral muscle $\mathrm{StO}_{2}$ in $\mathrm{PAH}$ with systemic oxygen delivery and an inverse correlation with systemic oxygen extraction rate, and b) they strengthen further the value of $\mathrm{StO}_{2}$ as a qualitative, noninvasive marker of $\mathrm{SvO}_{2}$, thus laying the foundation for further use of NIRS in the investigation of the pathophysiology of PAH. The absence of exercise data does not, of course, allow for complete extension of these results; however, our findings are still sufficient to support the use of NIRS in PAH, where routine patient assessment with right-heart catheterisation and $\mathrm{SvO}_{2}$ sampling is most often undertaken under resting conditions.

Marios Panagiotou ${ }^{1}$, Ioannis Vogiatzis ${ }^{2}$, Zafeiris Louvaris $^{2}$, Geeshath Jayasekera ${ }^{1}$, Alison McKenzie ${ }^{1}$, Neil Mcglinchey ${ }^{1}$, Julien S. Baker ${ }^{3}$, Alistair C. Church ${ }^{1}$, Andrew J. Peacock ${ }^{1}$ and Martin K. Johnson ${ }^{1}$

${ }^{1}$ Scottish Pulmonary Vascular Unit, Golden Jubilee National Hospital, Glasgow, UK. ${ }^{2}$ Faculty of Physical Education and Sports Sciences, National and Kapodistrian University of Athens, Athens, Greece. ${ }^{3}$ Institute of Clinical Exercise and Health Science, University of the West of Scotland, Hamilton, UK.

Correspondence: Marios Panagiotou, Scottish Pulmonary Vascular Unit, Golden Jubilee National Hospital, Agamemnon Street, Glasgow, G81 4DY, UK. E-mail: mariopanag@gmail.com

Received: Nov 032016 | Accepted: Nov 092016

Support statement: Marios Panagiotou is the recipient of an ERS PAH Long-Term Research Fellowship, number 2014-3106, supported by an unrestricted grant from GSK. Funding information for this article has been deposited with the Open Funder Registry.

Conflict of interest: Disclosures can be found alongside this article at erj.ersjournals.com

\section{References}

1 Panagiotou M, Vogiatzis I, Louvaris Z, et al. Near infrared spectroscopy for the assessment of peripheral tissue oxygenation in pulmonary arterial hypertension. Eur Respir J 2016; 48: 1224-1227.

2 Naeije R. Breathing more with weaker respiratory muscles in pulmonary arterial hypertension. Eur Respir J 2005; 25: 6-8.

3 DeLorey DS, Kowalchuk JM, Paterson DH. Relationship between pulmonary $\mathrm{O}_{2}$ uptake kinetics and muscle deoxygenation during moderate-intensity exercise. J Appl Physiol (1985) 2003; 95: 113-120.

4 Panagiotou M, Peacock AJ, Johnson MK. Respiratory and limb muscle dysfunction in pulmonary arterial hypertension: a role for exercise training? Pulm Circ 2015; 5: 424-434.

5 Dimopoulos S, Tzanis G, Manetos C, et al. Peripheral muscle microcirculatory alterations in patients with pulmonary arterial hypertension: a pilot study. Respir Care 2013; 58: 2134-2214.

6 Bradley EC, Barr JW. Determination of blood volume using indocyanine green (cardio-green) dye. Life Sci 1968; 7: 1001-1007.

7 Vogiatzis I, Habazettl H, Louvaris $\mathrm{Z}$, et al. A method for assessing heterogeneity of blood flow and metabolism in exercising normal human muscle by near-infrared spectroscopy. J Appl Physiol (1985) 2015; 118: 783-779.

8 Habazettl H, Athanasopoulos D, Kuebler WM, et al. Near-infrared spectroscopy and indocyanine green derived blood flow index for noninvasive measurement of muscle perfusion during exercise. J Appl Physiol (1985) 2010; 108: 962-967. 
9 Guenette JA, Vogiatzis I, Zakynthinos S, et al. Human respiratory muscle blood flow measured by near-infrared spectroscopy and indocyanine green. J Appl Physiol (1985) 2008; 104: 1202-1210.

10 Louvaris Z, Kortianou EA, Spetsioti S, et al. Intensity of daily physical activity is associated with central hemodynamic and leg muscle oxygen availability in COPD. J Appl Physiol (1985) 2013; 115: 794-802.

Copyright @eERS 2017 